\title{
Ruhe, bitte!
}

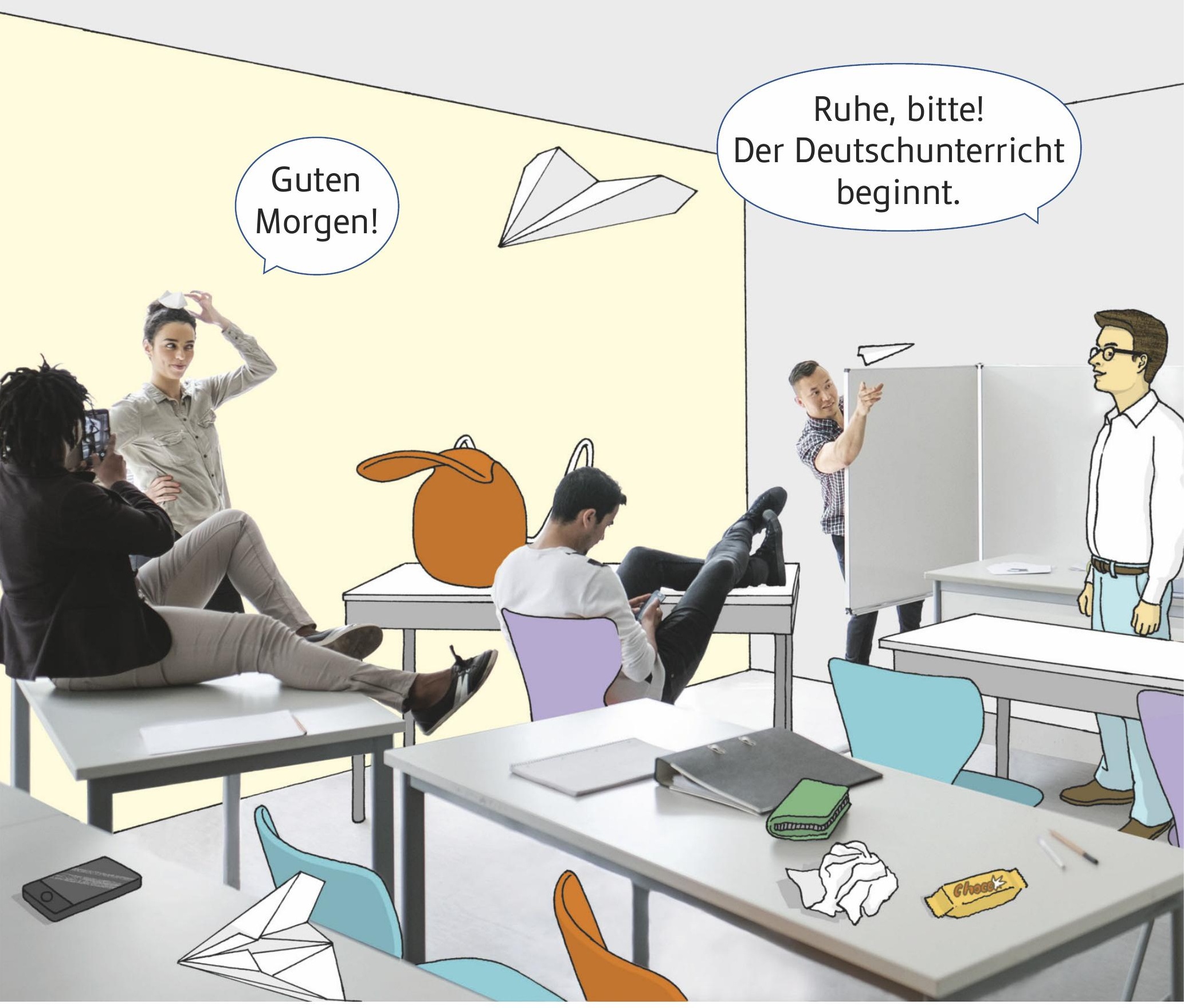

1 Was siehst du? Sprich.

2 Höre und sprich nach. (3) (8) 1

\section{Ruhe}


Ruhe, bitte!

3a Sprich nach und fahre mit dem Finger nach. (2) (1)
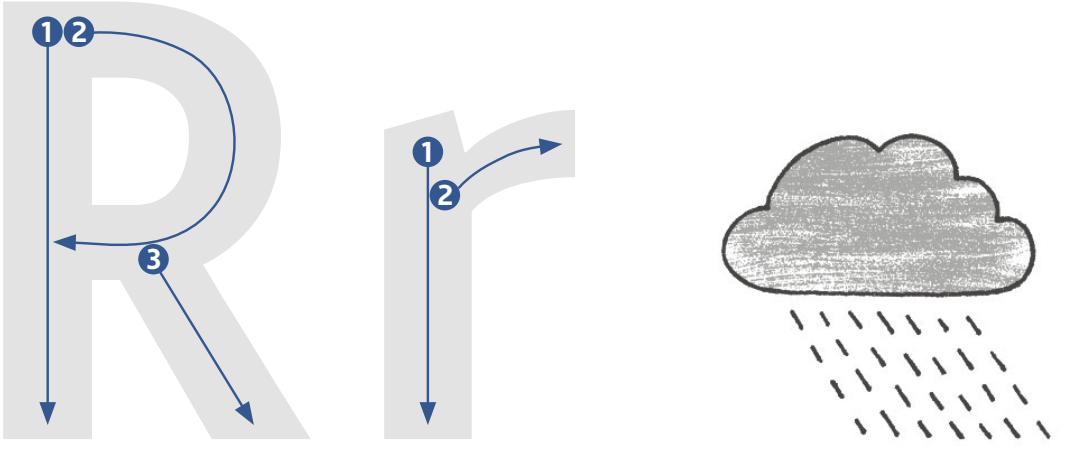

3b Schreibe.

$\mathrm{R}$

$r$

R r

Regen

4 Lies. 영

ra sa na da na sa ar as an ad an as re se ne de ne se er es en ed en es

5 Markiere R r.

$\begin{array}{lllllllllllllllllllll}R & H & N & F & r & f & o & a & l & i & r & R & Y & H & R & A & r & c & g & r & t\end{array}$ Radio Arm Ruhe wir wer Lehrer Schüler 
6a Höre und sprich nach. (2) (1) 12
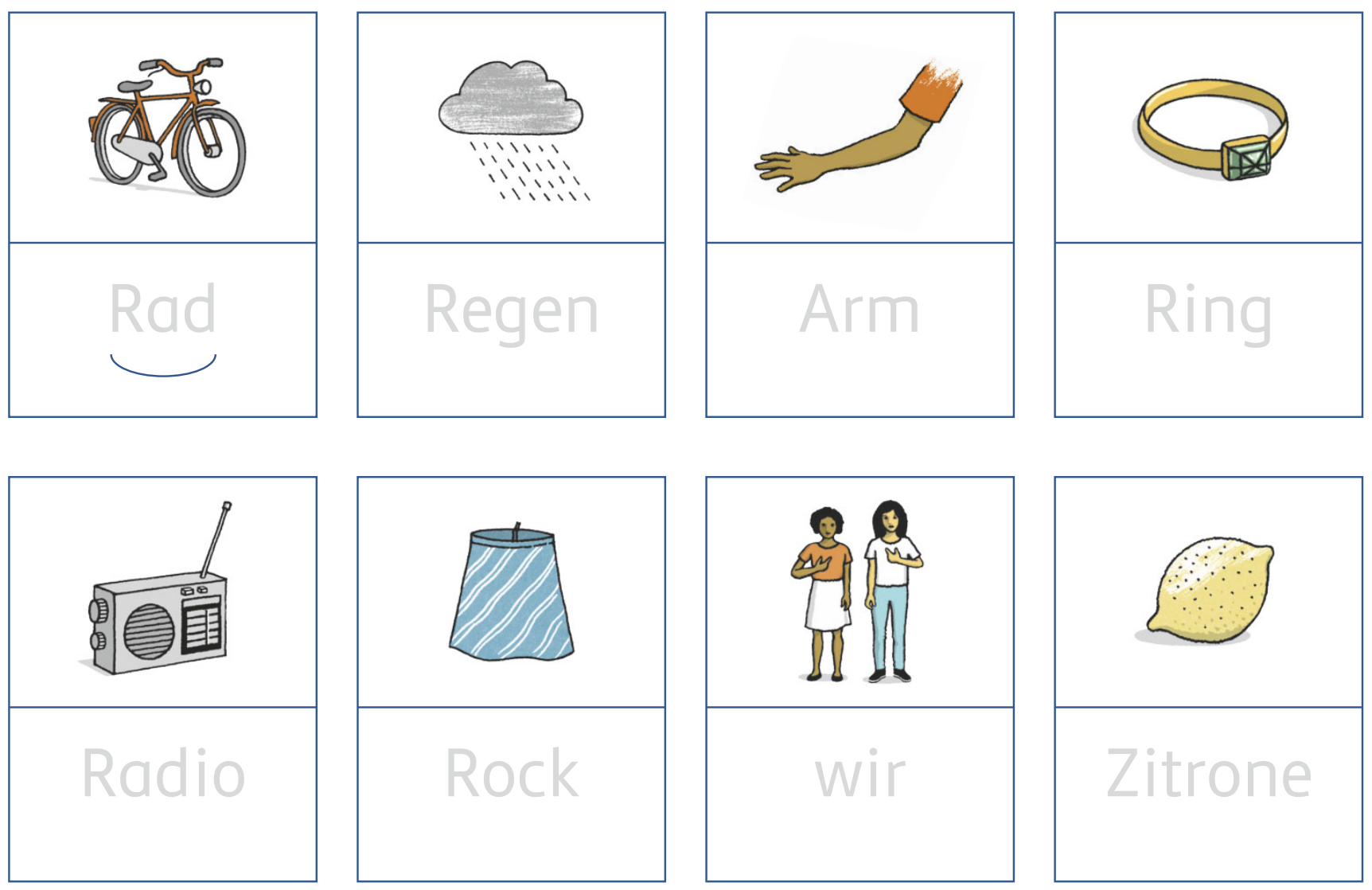

6b Zeichne die Silbenbögen ein.

6c Schreibe R r oder das ganze Wort nach.

6d Lies und schreibe den Buchstaben in die Luft. 응
A wie Ananas
D wie Dose
S wie Salat
$\mathrm{N}$ wie Nase
E wie Elefant
$\mathrm{R}$ wie Regen 


\section{Ruhe, bitte!}

7 In welchem Wort ist R r? Verbinde.

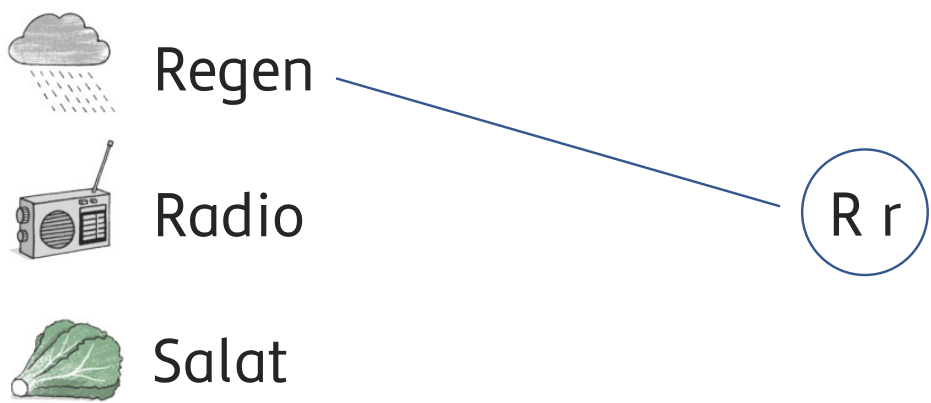

Nase

Arm

Ring

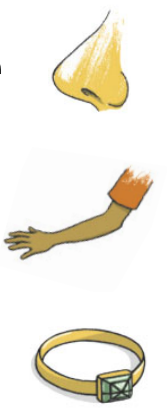

8a Male.
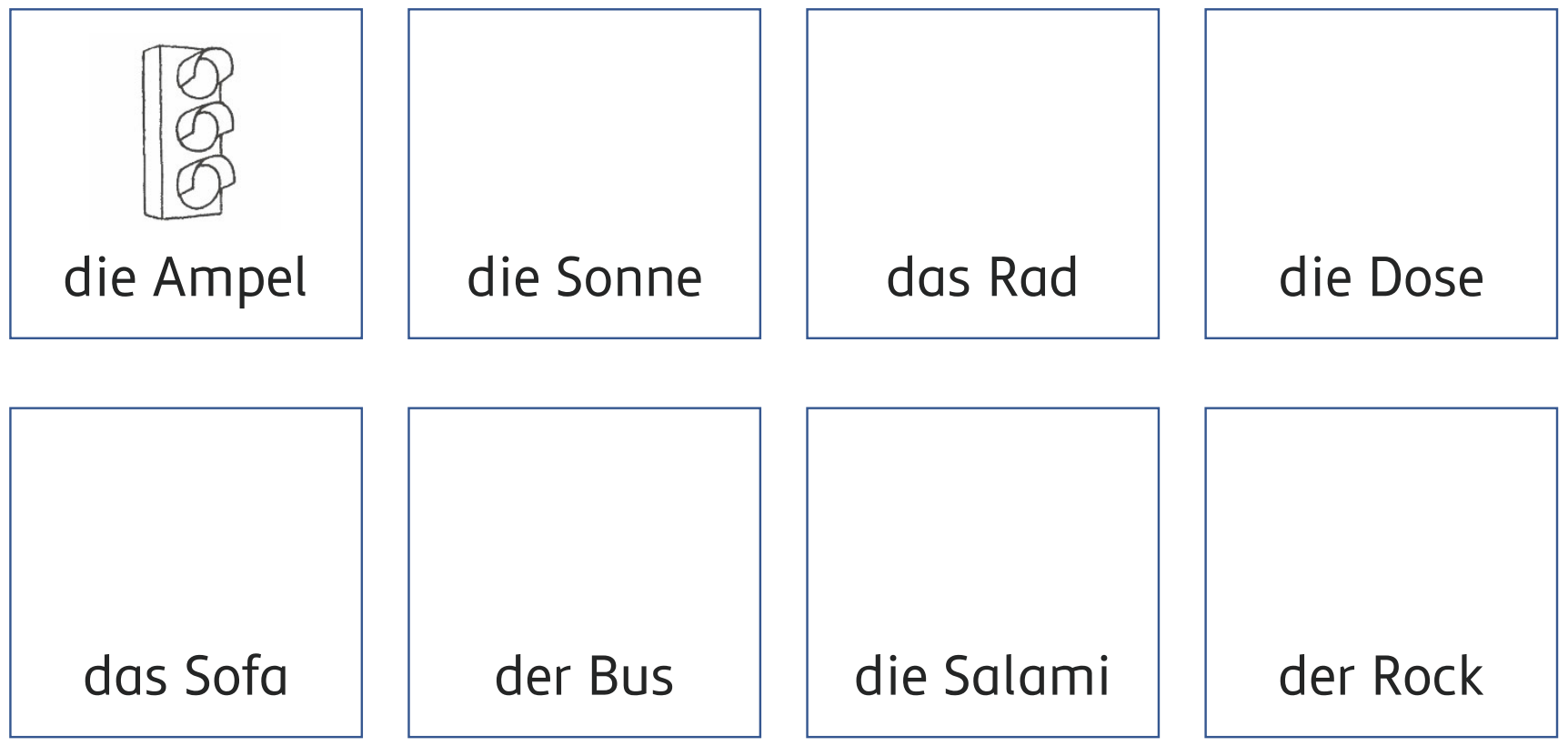

8b Malt und ratet an der Tafel.

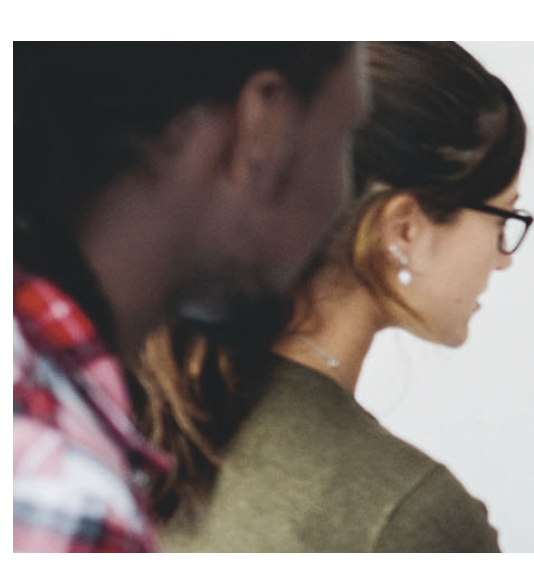

\section{Suppe?}

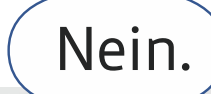

Nein.

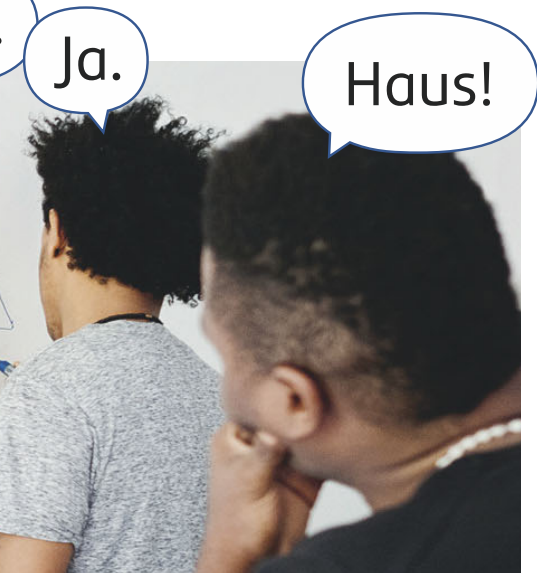


9a Was ist wichtig? Sprecht in der Klasse.
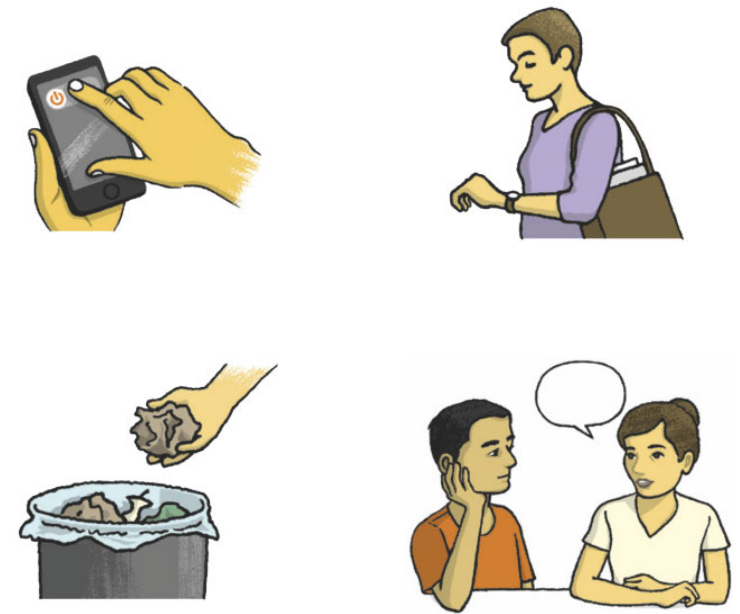
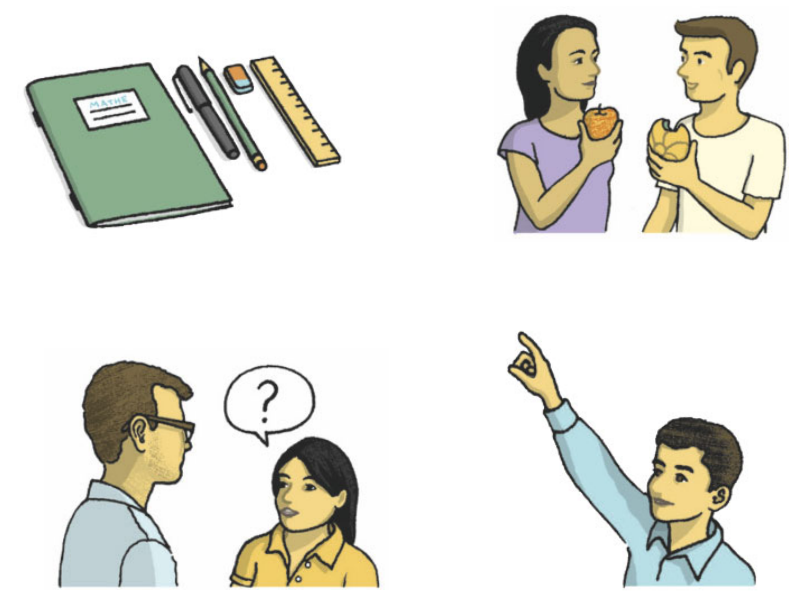

9b Klatsche und sprich mit. (5) (-) ${ }_{13}$

Im Leben gibt es Regeln, die Schule hat sie auch. Wir lernen unsere Regeln, weil jeder Regeln braucht.

Ich komme nicht zu spät. Spät kommen, das geht nicht! Lieber bin ich pünktlich.

- Ja, ja wunderbar!

Ich bin nicht zu laut. Nein, nein, das geht nicht! Besser bin ich leise. - Ja, ja wunderbar!

Der Müll liegt auf dem Boden. Nein, nein, das geht nicht! Ich schmeiße ihn in den Eimer. - Ja, ja wunderbar!
Ich vergess' die Hausaufgaben. Nein, nein, das geht nicht! Die Hausaufgaben muss ich machen. Ja, ja wunderbar!

Ich spiele mit dem Handy. Auf keinen Fall, das geht nicht! Das Handy bleibt aus. - Ja, ja wunderbar!

Die Regeln sind ganz einfach. Wir merken sie uns fest, Jetzt können wir besser lernen, weil lernen wichtig ist.

Sei zu mir, wie zu dir! Ich bin zu dir, wie zu mir!

Sind jetzt alle Regeln klar? Ja, ja wunderbar! 


\section{Ruhe, bitte!}

10 Schreibe die Wörter aus 6a und 7.

11a Höre und lies mit. (5) (1) ${ }_{14}$ 国

- Hallo. Ich bin neu in der Klasse. Wie heißt du?

- Ich heiße Sara. Und du?

- Ich heiße Andreas. Und wer ist das?

- Das ist Herr Eder. Er ist unser Lehrer.

Er ist sehr nett. Psst, Ruhe, bitte.

Der Unterricht beginnt.

11b Schreibe die Antwort.

Wie heißt der Lehrer?

\section{Sprich. 8}

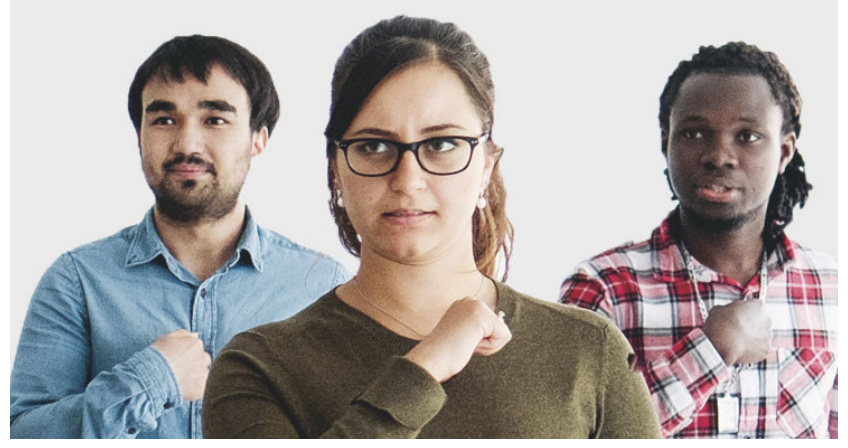

Stehe auf und höre. Der Lehrer oder die Lehrerin stellt eine Frage. Antworte: Ich, Du oder Wir.

Zeige dabei auf dich, auf einen Mitschüler oder eine Mitschülerin oder auf die ganze Klasse. 
13a Höre und lies mit. (3) (9)

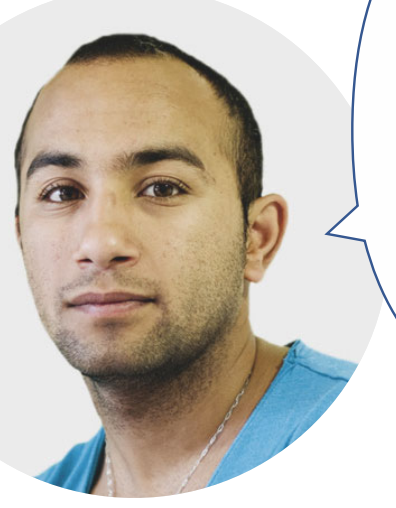

Ich heiße Saad.

Wie geht es dir? Mir geht es gut.

Das ist meine
ufgabe für dich.
Bis bald.

Das ist meine
Aufgabe für dich.
Bis bald.

Das ist meine für d
Bis bald.

13b Meine Aufgabe für dich. Markiere R r.
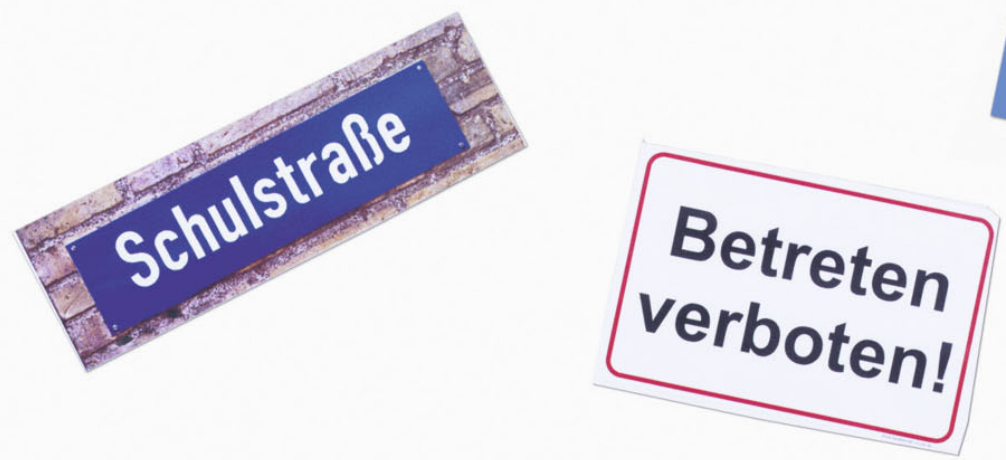

Hallo.

r. 2 
T. Meine Lernseiten

$\star$ Lies. 目

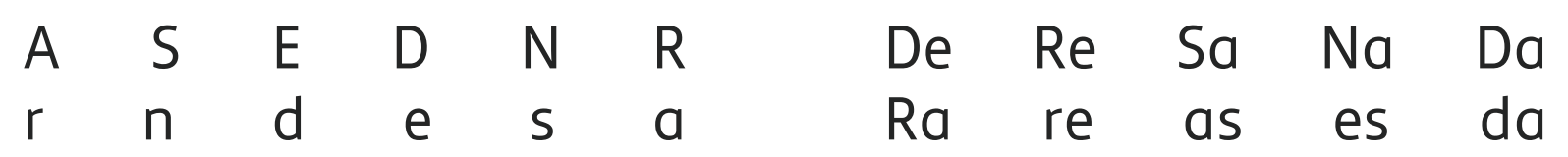

$\star \star$ Verbinde.
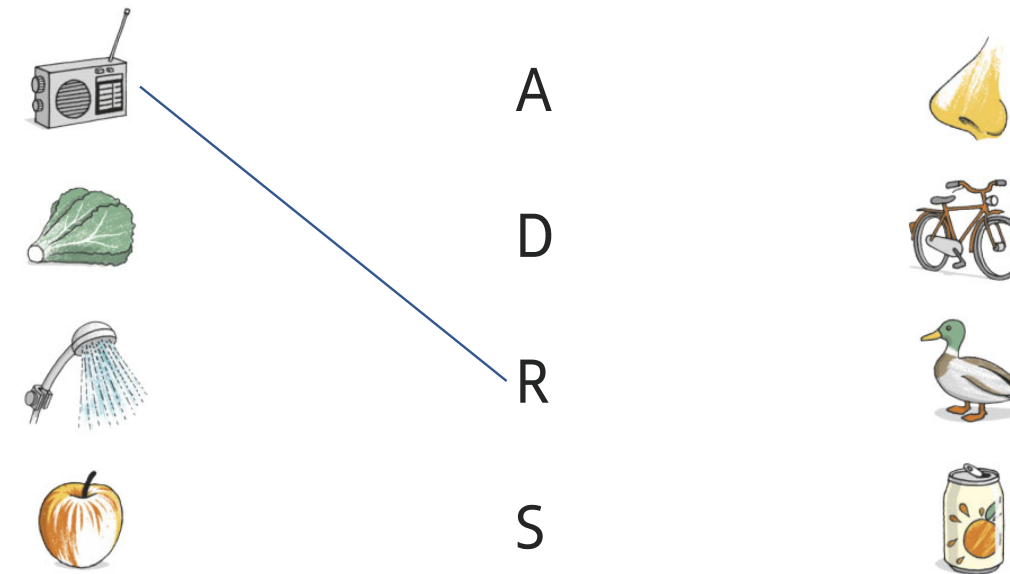

E

D

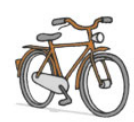

$\mathrm{N}$

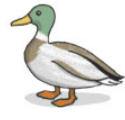

D

S

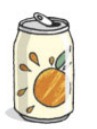

$\mathrm{R}$

$\star \star \star$ Male drei Regeln für die Schule.
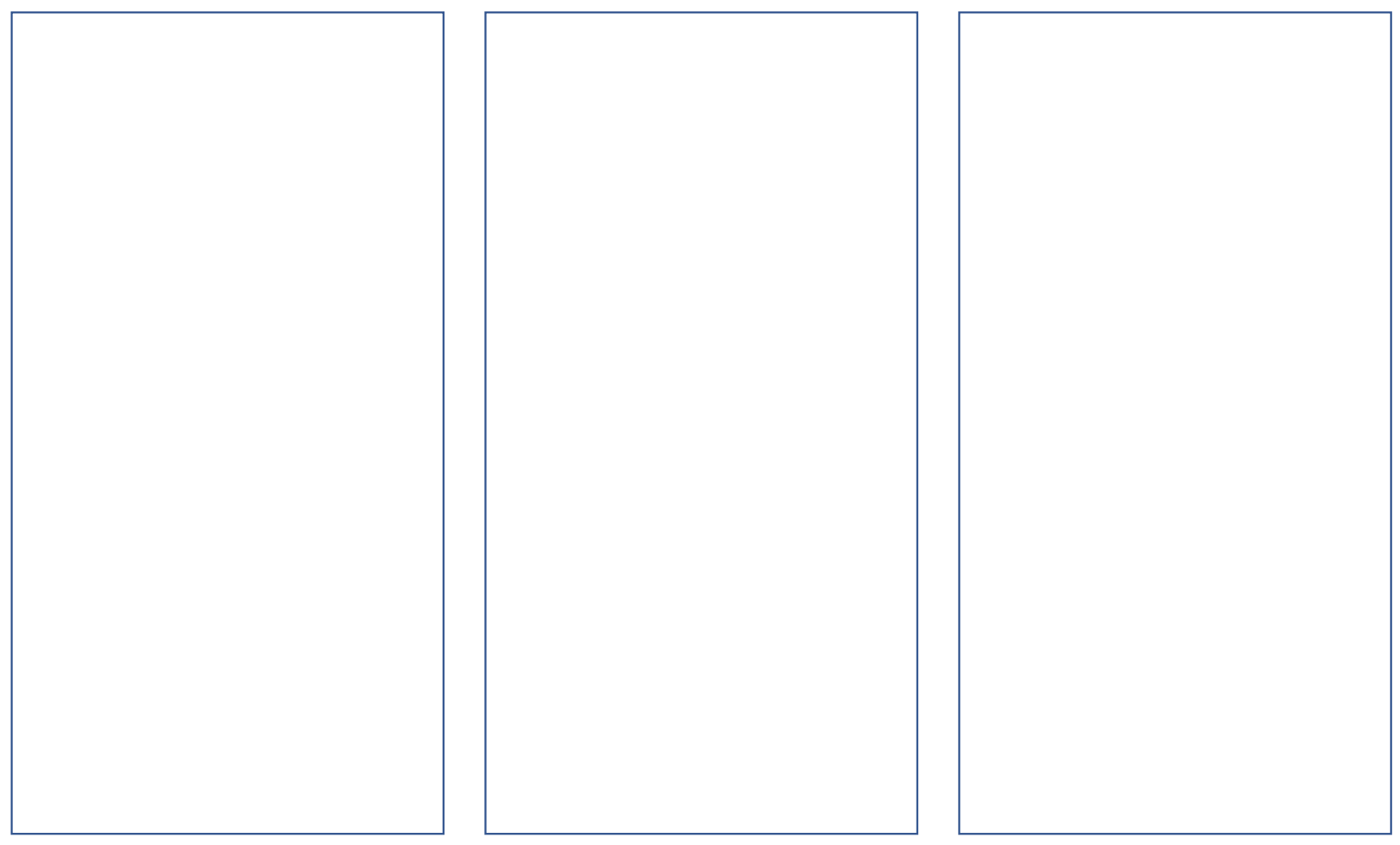
$\star$ Welche Buchstaben kennst du? Markiere.

Aa Ff Bb Ss LI Dd RrOoKk Ee Nn Zz Ii

$\star \star$ Male.
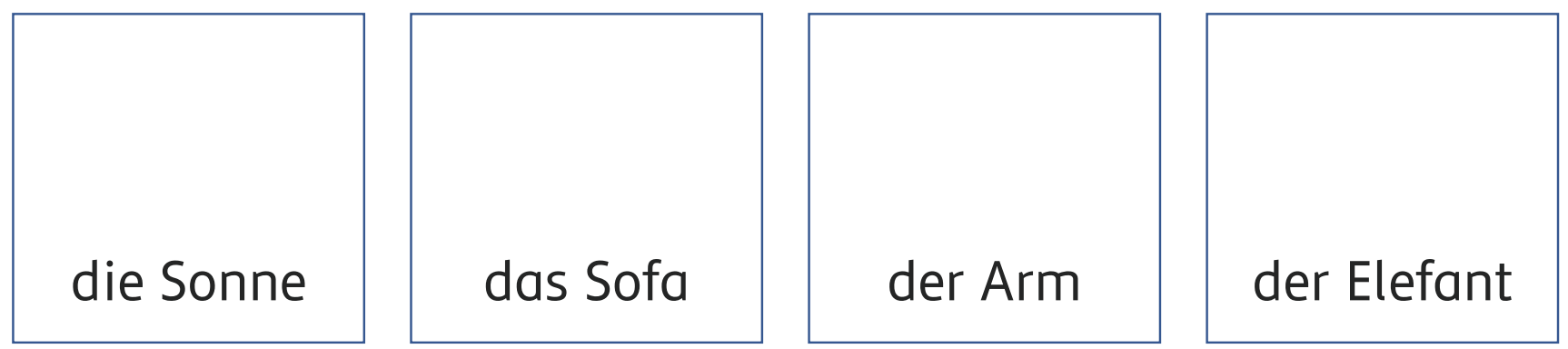

$\star \star \star$ Was fehlt? Schreibe.

- Guten Morgen!

Mein ist Frau Peters.

Ich bin

Wie heißt du?

- Ich heiße

- Ich komme aus Deutschland. Woher kommst du?

- Ich komme aus

- Super. Auf Wiedersehen! 
专 Mein Wortschatz

Schreibe.

der Arm

das Rad

rel das Radio

der Regen

der Ring

der Rock

die Ruhe

der Schüler

der Lehrer

Lies und sprich.

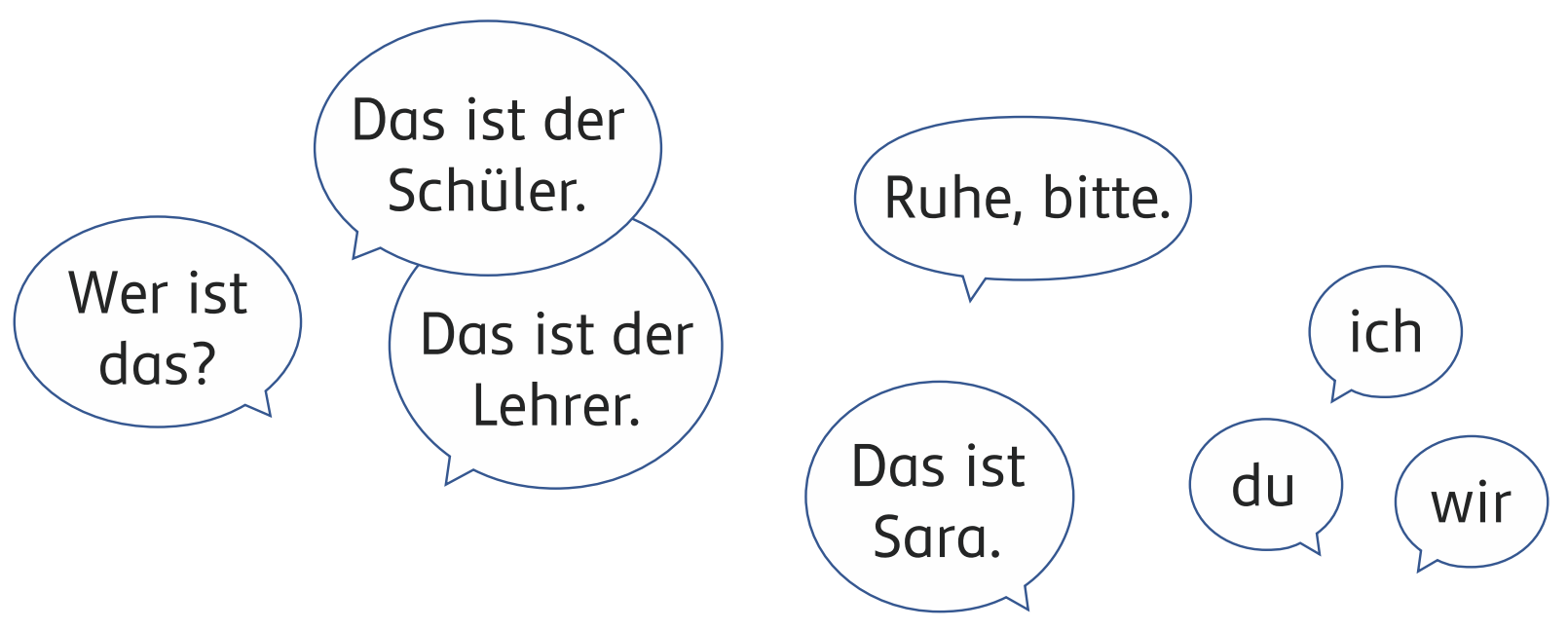




\section{Lernen unterwegs}

Lerne die Wörter.

der Apfel

die Ananas

der Regen

der Schüler, die Schülerin

das Sofa

der Salat

die Salami

der Ring

die Ente

der Elefant

das Radio

das Essen

die Dose

Deutschland

das Datum

der Lehrer, die Lehrerin 
Open Access Dieses Kapitel wird unter der Creative Commons Namensnennung - Nicht kommerziell - Keine Bearbeitung 4.0 International Lizenz (http://creativecommons.org/licenses/by-nc-nd/4.0/deed.de) veröffentlicht, welche die nicht-kommerzielle Nutzung, Vervielfältigung, Verbreitung und Wiedergabe in jeglichem Medium und Format erlaubt, sofern Sie den/die ursprünglichen Autor(en) und die Quelle ordnungsgemäß nennen, einen Link zur Creative Commons Lizenz beifügen und angeben, ob Änderungen vorgenommen wurden. Die Lizenz gibt Ihnen nicht das Recht, bearbeitete oder sonst wie umgestaltete Fassungen dieses Werkes zu verbreiten oder öffentlich wiederzugeben.

Die in diesem Kapitel enthaltenen Bilder und sonstiges Drittmaterial unterliegen ebenfalls der genannten Creative Commons Lizenz, sofern sich aus der Abbildungslegende nichts anderes ergibt. Sofern das betreffende Material nicht unter der genannten Creative Commons Lizenz steht und die betreffende Handlung nicht nach gesetzlichen Vorschriften erlaubt ist, ist auch für die oben aufgeführten nicht-kommerziellen Weiterverwendungen des Materials die Einwilligung des jeweiligen Rechteinhabers einzuholen. 